\title{
Supra-Acetabular Pelvic External Fixator- is it safe?
}

\author{
Elaine Soh Zi Fan*, Rizal Abdul Rani and Noor Hamdan Mohamad Yahaya \\ Department of Orthopaedic and Traumatology, National University of Malaysia, Malaysia
}

Received: February 18, 2018; Published: March 05, 2018

*Corresponding author: Elaine Soh Zi Fan, Department of Orthopaedic and Traumatology, National University of Malaysia, Level 9, Department of Orthopaedic, Hospital University Kebangsaan Malaysia, Jalan Yaacob Latiff, 56000 Bandar Tun Razak, Cheras, Kuala Lumpur, Malaysia, Tel: +60127742313 ;Email: esssi@hotmail.com

\begin{abstract}
Pelvic external fixator is used for stabilisation of pelvic fracture. Iliac crest placement of pin is commonly used but supra-acetabular pin placement is becoming more popular as it can offer more stability, avoiding irritation to abdominal soft tissue and prevent disruption to the skin over iliac crest especially if anterior approaches to the sacroiliac joint are planned for future surgery. However, there is risk of hip septic arthritis due to proximity of the pin to the hip capsule but cases are rarely reported. This case report highlights the importance of correct placement of pin and the possibilities of hip septic arthritis as a complication of supra-cetabular pin.
\end{abstract}

Keywords: Supra-Acetabular Pin; Septic Arthritis

\section{Introduction}

Pelvic external fixator is commonly used for temporary or definitive stabilisation of pelvic fractures. It can be classified based on the orientation of the direction of pin purchase, either anterosuperior (transiliac crest) or anteroinferior (supraacetabular). Anterosuperior pin placement is easier and widely used with less risk of neurovascular injury [1]. Pin site infection rate of pelvic external fixator ranges from 13-50\% [2]. One of the potential complications by supra-acetabular pin placement is hip penetration resulting in septic arthritis [3]. However, cases are rarely reported. We present a case of hip septic arthritis which likely occurred secondary to the penetration of the supra-acetabular pin into the hip joint.

\section{Case Report}

A 19-years-old gentleman was involved in a motor vehicle accident where he sustained polytrauma with pelvic ring disruption (Tile Type C). He was managed primarily at a general specialist hospital with supraacetabular external fixator under emergency setting for stabilisation of the pelvic fracture. At 6 weeks post operation, the fracture had stabilised and pins are removed. He was then started on progressive weight bearing and ambulation. After removal of external fixator, he noted pus discharge from the pin tract site but did not seek further medical advice from the hospital. He continues with self dressing with normal saline and oral antibiotic for 6 weeks duration by the general practitioner. During the subsequent follow up with the orthopaedic team, there were no more pus discharge from pin tract and he was prescribed with another 6 weeks course of oral antibiotic. The pin tract eventually healed. He presented to our centre 9 months after the injury complaining of persistent pain over right hip and shortening of right lower limb, with antalgic gait. He denies any fever or discharge from the hip wound. Clinically noted there is healed stab wound scar with no sign of local 3 infections. There is shortening of $2 \mathrm{~cm}$ on the right lower limb with limited range of motion of right hip due to pain. He ambulates with auxiliary crutches. Infective markers were raised (WBC 8.1 x 109/L, CRP $8.15 \mathrm{mg} / \mathrm{dl}$, ESR 88mm/hr.)

Radiographs of right hip noted showed subchondral sclerosis of right hip joint, with reduction in joint space and irregular joint surfaces (Figure 1). Subsequently, we performed an MRI of right hip joint which showed destructive changes at the articular surface of right hip joint involving the acetabulum, femoral head and articular cartilage suggestive of septic arthritis with surrounding muscle and intramuscular inflammation. Needle biopsy of right hip under ultrasound guidance was carried out. There was no effusion and tissue histopathology showed fibrocartillagenous tissue with scattered vessels and mild lymphocytes and plasma cell infiltrate [4]. The hip tissue culture yields no growth. He was treated with 6 weeks of oral antibiotic (Rifampicin and fusidic acid) and the symptoms and clinical infective markers showed improvement (CRP 5.46mg/dl, ESR 87mmlhr) (Figure 2). At 6 months follow up, patient has improved and was able to weight bear without pain, with infective markers remained static (CRP $6.17 \mathrm{mg} / \mathrm{dl}$, ESR 85mmlhr). 


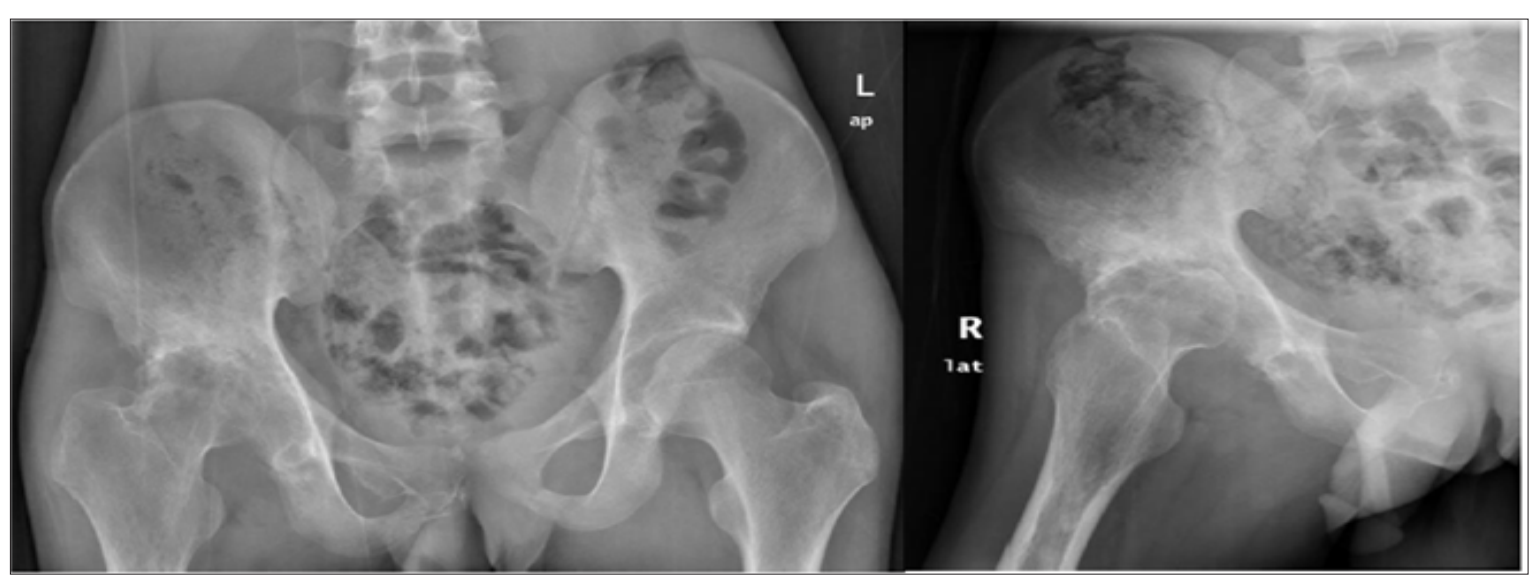

Figure 1: Plain radiographs showing sclerotic changes in the right hip with reduction in joint space and irregular joint surface.

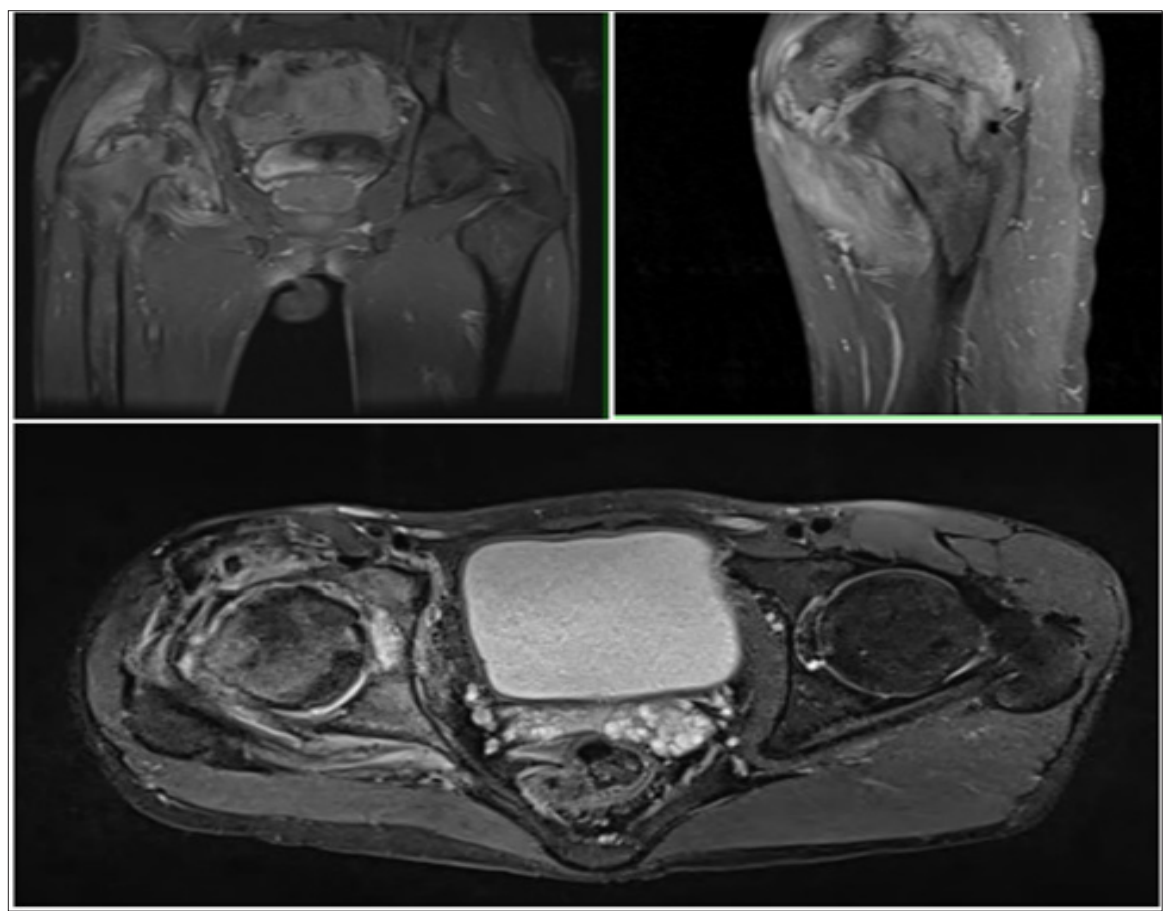

Figure 2: MRI of the right hip joint showing destructive changes at the articular surface with inflammation suggestive of septic arthritis.

\section{Discussion}

Modern pelvic external fixation is classified based on the orientation of the direction of pin purchase, either anterosuperior (transiliac crest) or anteroinferior (into the supraacetabular dense bone). Anterosuperior pin placement is relatively easier and widely used with less risk of neurovascular injury. However, due to the iliac wing being curved and narrow, there is limitation of additional pins insertion for firmer purchase. Moreover, less density of bone is available for anterosuperior pin purchase, hence fracture reduction using joystick maneuver is 5 not advisable due to bone pin interface fatigues [1]. Kim et al found anteroinferior pin placement offered greater fracture stability. It showed lower sacroiliac displacement in Tile Type B1 and C injuries [5]. Futhermore, supracetabular frames are oriented below and away from the abdomen unlike the iliac crest frame, resulting in less irritation to anterolateral abdominal soft tissues. It also prevents disruption to the skin over iliac crest if anterior approaches to the sacroiliac joint are planned for future surgery [5]. Despite the advantages of supracetabular pin, one of the potential complications is hip penetration resulting in septic arthritis [3].

Mason et al found that the overall complication rate of pelvic external fixation is high and having marked difference between temporary (21\%) and definitive fixators (62\%). Pin site infection rate in temporary fixators is $13 \%$ while definitive fixators are 50 $\%$ [2]. Cases of septic arthritis of hip secondary to supracetabular pin are rarely reported. Kyle et al reported a similar case of septic arthritis of hip due to supracetabular pin insertion for stabilisation of pelvic ring disruption. In this case, superior ramus screw was also 
placed. Post operative radiographs noted metal debris proximal to the acetabulum where the anterior supraacetabular half pin came into contact with the superior ramus screw. They explained that abberant pin trajectory and intraarticular penetration during attempts to place the pin might have cause the septic arthritis [4].

Surgical technique of supraacetabular external fixator has been well described. Cadaveric anatomical study on supraacetabular pin placement has also been carried out by Haidukewych et al. using fluoroscopic guidance; all pins were placed extra capsular in this study. The average distance that the capsule of the hip inserted above the joint was $16 \mathrm{~mm}$ while the average pin was placed $20 \mathrm{~mm}$ above the joint. It showed that supraacetabular pin can be placed accurately percutaneously. They also found that mobilisation of patient were easier with supracetabular frames, and patient able to sit upright in a chair, but hip flexion more than 95 degree is difficult [5].Review article by Stavlas also recommended an insertion point $2 \mathrm{~cm}$ above the hip joint to avoid capsule penetration [3].

\section{Conclusion}

Septic arthritis should be cautioned as a possible complication of supraacetabular pin and fluoroscopic guidance insertion is recommended.

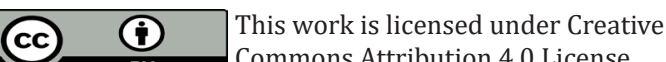

Submission Link: http://biomedres.us/submit-manuscript.php

\section{References}

1. Kim WY, Trevor C Hearn, Osama Seleem, Essai Mahalingam, David Stephen, et al. (1999) Effect of pin location on stability of pelvic external fixation. Clinical Orthopaedics and related research 361: 237-244.

2. Mason WTM, Khan SN, James CL, Chesser TJS, Ward AJ (2005) Complications of temporary and definitive external fixation of pelvic ring injuries. Injury Int J Care Injured 36(5): 599-604.

3. Stavlas P, Polyzois D (2005) Septic arthritis of the major joints of the lower limb after periarticular external fixation application: are conventional safe corridors enough to prevent it? Injury Int J Care Injured 36(2): 239-247.

4. Kyle T Judd, Todd O McKindley (2009) Septic arthritis of the hip associated with supracetabular external fixation of unstable pelvic ring: a case report. Iowa Orthopaedics Journal 29: 124-126.

5. Haidukewych, Sanjay Kumar, Branko Prpa (2003) Placement of halfpins for supra-acetabular external fixation: An anatomic study. Clinical Orthopaedics and related research 411: 269-273.

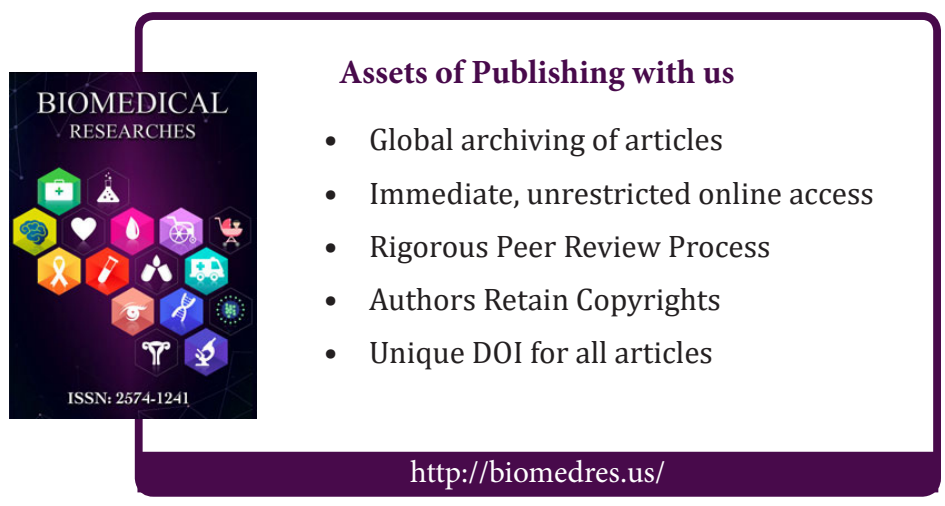

